Für konkrete Anhaltspunkte, die in einem Arzthaftungsverfahren Zweifel an der erstinstanzlichen Beweiswürdigung wecken sollen, reicht dies indes nicht aus. Erforderlich ist vielmehr, dass der Patient entweder ein Privatgutachten vorlegt, zumindest aber selbst medizinische Fundstellen oder Leitlinien benennt, die für seine Behauptung streiten. Wird ein solches Privatgutachten nicht vorgelegt und fehlt es auch im Übrigen an Anhaltspunkten dafür, dass das Gutachten in sich widersprüchlich oder der Sachverständige erkennbar nicht sachkundig ist, kommt eine Wiederholung der Beweisaufnahme nicht in Betracht (Senat, Beschl. v. 15.5.2018 - 4 U 750/17 -, juris). Gleiches gilt für den Vorwurf des Kl., bei der erneuten Wiedervorstellung am 15.12.2014 (...) sei keine adäquate Behandlung der Narbenhernie, insbesondere keine Verschreibung eines Bruchbandes zur Beschwerdelinderung erfolgt. Auch insoweit hat der Sachverständige die Empfehlung, sich in der viszeralchirurgischen Sprechstunde vorzustellen als adäquat bezeichnet, da die Narbenhernie asymptomatisch war. Schmerzen, die einen Leidensweg begründen könnten, werden vom Kl. nicht dargestellt. Hinzu kommt, dass nach dem eigenen Vortrag des Kl. der Zeitpunkt der Operation am 30.3.2015 auf die Einnahme von Blutverdünnern (erforderlich wg. Thrombose) zurückzuführen ist, die ein Herzspezialist erst einstellen musste. Ein Kausalzusammenhang mit Maßnahmen, die die Bekl. hätte veranlassen müssen, besteht auch aus diesem Grund nicht.

[10] Der Senat rät auf dieser Grundlage zu einer Berufungsrücknahme, die zwei Gerichtsgebühren spart. Der Streitwert für das Berufungsverfahren soll auf 30.893,88 EUR festgesetzt werden.

https://doi.org/10.1007/s00350-020-5764-z

\section{Anmerkung zu OLG Dresden, Beschl. v. 16.3.2020 - 4 U 2626/19 (LG Leipzig)}

\section{Claudia Achterfeld}

Von Notfällen abgesehen, muss dem Patienten ausreichend Zeit verbleiben, das Für und Wider der vorgeschlagenen Therapie abzuwägen und sich gegebenenfalls auch mit Dritten besprechen zu können. Entscheidend für die Frage des richtigen Aufklärungszeitpunkts sind die Umstände des Einzelfalls, insbesondere die Schwere des Eingriffs und die mit ihm einhergehenden Risiken ${ }^{1}$. Pauschale Fristen für die Zeitspanne zwischen Aufklärung, Einwilligung und Eingriff sind damit nicht zu vereinbaren ${ }^{2}$. Ist ein operativer Eingriff mit einem stationären Aufenthalt verknüpft, fordert die Judikatur mitunter eine Aufklärung bereits bei Festlegung des Operationstermins ${ }^{3}$, spätestens aber am Vortag der Operation, sofern sie hier zu einem Zeitpunkt erfolgt, die dem Patienten die Wahrung seines Selbstbestimmungsrechts erlaubt ${ }^{4}$. Das wird bei einer Aufklärung über gravierende Risiken am Vorabend der Operation regelmäßig nicht mehr der Fall sein ${ }^{5}$. Auch bei rein kosmetischen Operationen wird man dem Patienten einen längeren Entscheidungszeitraum zubilligen müssen ${ }^{6}$. Bei ambulanten oder rein diagnostischen (Routine-)Eingriffen mit geringen und wenig einschneidenden Risiken erachtet die Rechtsprechung eine Aufklärung am Operationstag selbst dagegen in der Regel für ausreichend $^{7}$. Der Patient stehe hier regelmäßig nicht so stark unter dem Druck des „Eingebundenseins“ in den Krankenhausbetrieb $^{8}$. Letztlich entscheidend für die Frage der Rechtzeitig-

Rechtsanwältin Dr. iur. Claudia Achterfeld, Wiss. Mitarb.,

Institut für Medizinrecht,

Universität zu Köln,

Albertus-Magnus-Platz, 50923 Köln, Deutschland keit der Aufklärung muss aber auch bei ambulanten Eingriffen die Risikoneigung eines Eingriffs bleiben.

Durch die Entwicklung minimal-invasiver Verfahren und kurzwirksamer Anästhetika können heute sogar neurochirurgische Eingriffe wie etwa Bandscheiben-Operationen oder auch herzchirurgische Eingriffe wie etwa Herzschrittmacher-Implantationen ambulant erfolgen ${ }^{9}$. Tatsächlich werden derzeit bereits $37 \%$ der Operationen in Deutschland unter ambulanten Voraussetzungen durchgeführt, Tendenz steigend ${ }^{10}$. Dass ein Eingriff ambulant durchgeführt wird, kann daher stets nur Indikator für ein geringes Risikospektrum sein. Gehen mit einem ambulanten oder auch rein diagnostischen Eingriff erhebliche Risiken einher, ist eine frühere Aufklärung erforderlich ${ }^{11}$. Voraussetzung für die Aufklärung am Operationstag ist überdies, dass die Aufklärung nicht erst so unmittelbar vor dem Eingriff erfolgt, dass der Patient unter dem Eindruck steht, sich nicht mehr aus einem bereits in Gang gesetzten Geschehensablauf lösen zu können ${ }^{12}$. Dem Patienten muss auch hier die Gelegenheit zu einem ruhigen Abwägen verbleiben. Ob vor diesem Hintergrund die Aufklärung zu einer ambulant durchzuführenden Koloskopie nach der erforderlichen abgeschlossenen Darmreinigung mit dem OLG Dresden ernsthaft für rechtzeitig erachtet werden kann, erscheint mehr als fragwürdig. Da der Patient im Vorfeld jedoch bereits ordnungsgemäß aufgeklärt worden war, kam es auf die Aufklärung am Operationstag vorliegend aber auch nicht mehr an.

1) $\mathrm{BGH}, \mathrm{NJW}$ 1992, $2351 \mathrm{f} .=$ MedR 1992, 277; vgl. auch BTDr. 17/10488, S. 25; Achterfeld, in: Narr (Begr.), Ärztliches Berufsrecht, 28. EGL 2018, D-VI 5., Rdnr. 32.

2) BT-Dr. 17/10488, S. 25.

3) BGH, NJW 1992, 2351, $2352=$ MedR 1992, 277; NJW 2003, 2012, 2013 = MedR 2003, 576; zu Auswüchsen krit. Katzenmeier, in: Laufs/Katzenmeier/Lipp, Arztrecht, 8. Aufl. 2020, Kap. V, Rdnr. $93 \mathrm{f}$.

4) $\mathrm{BGH}$, NJW 2003, 2012, 2013 = MedR 2003, 576; OLG Hamm, MedR 2011, 439, 440.

5) BGH, NJW 1998, 2734 „,Tumoroperation“; OLG Köln, VersR 2012, 863 „Mitralklappenoperation“.

6) Spindler, in: BeckOGK, Stand 1.8.2020, $\$ 823, \mathrm{Rdnr} .840$

7) BGH, NJW 1994, 3010, $3011=$ MedR 1995, 20; OLG Koblenz, NJW-RR 2002, 816, 818

8) BGH, NJW 1994, 3010, $311=$ MedR 1995, 20.

9) Vgl. Übersicht bei Bundesverband für ambulantes Operieren, e. V., https://www.operieren.de/e3224/e10/e589/, zuletzt abgerufen am 31.8.2020.

10) Bundesverband für ambulantes Operieren, e.V., https://www. operieren.de/e3224/e308/e309/, zuletzt abgerufen am 31.8.2020.

11) BGH, NJW 1994, 3010, 311 = MedR 1995, 20; vgl. auch Frahm Walter, Arzthaftungsrecht, 6. Aufl. 2018, Rdnr. 218 m.w. N.

12) $\mathrm{BGH}, \mathrm{NJW} 1994,2410,2411 ; 3010,3011=\operatorname{MedR} 1995,20$.

\section{Arzthaftung nach unterlassener Aufklärung werdender Eltern über mögliche Behinderung des ungeborenen Kindes}

BGB §§286, 288, 1612a, SchKG §12 Abs. 1, SGB X §116,

SGB XI §34, StGB §218a Abs. 2, ZPO §§92 Abs. 1, 100 Abs. 1, Abs. 4, 269, 286 Abs. 1, 511 ff. 529, 533, 708 Nr. 11, 711

Schadensersatzanspruch der Eltern bei unterlassenem Hinweis der Ärzte auf eine mögliche schwere Behinde-

Eingesandt von Prof. Dr. iur. Christian Katzenmeier, Köln; bearbeitet von Dipl. Wirtschaftsjuristin (FH) Anne Ziegler, LL.M. (Medizinrecht), Kanzlei Ziegler \& Kollegen, 35037 Marburg, Deutschland 